\title{
The Steiner Minimal Network for Convex Configurations
}

\author{
D. A. Thomas, J. H. Rubinstein, and T. Cole \\ Mathematics Department, Melbourne University, \\ Parkville, Victoria 3052, Australia \\ doreen $@$ mundoe.maths.mu.oz.au
}

\begin{abstract}
Suppose $X$ is a convex configuration with radius of maximum curvature $r$ and at most one of the edges joining neighboring points has length strictly greater than $r$. We use the variational approach to show the Steiner tree $S$ coincides with the minimal spanning tree and consists of all these edges with a longest edge removed. This generalizes Graham's problem for points on a circle, which we had solved. In addition we describe the minimal spanning tree for certain convex configurations.
\end{abstract}

\section{Introduction}

If $x_{1}, \ldots, x_{n}$ is a finite set of points in the Euclidean plane, the Steiner problem is to find the shortest network $S$ connecting the points. Melzak [3] has given an algorithm for finding $S$ but as $n$ increases the number of steps rise exponentially. The problem has been shown to be NP complete [1].

Graham [2] made an interesting conjecture, about 20 years ago, about $S$ in the case where all the points lie on a circle. We have shown the conjecture is true [7] and proved the following theorem.

Theorem 1. Suppose that $x_{1}, x_{2}, \ldots, x_{n}$ lie on a circle $C$ of radius $r$, with $x_{i-1}$ adjacent to $x_{i}, 2 \leq i \leq n$. If at most one of the edges $x_{i} x_{i+1}$ and $x_{n} x_{1}$ has length strictly greater than $r$, for $1 \leq i \leq n-1$, then the shortest network $S$ consists of all the edges $x_{i} x_{i+1}$ plus $x_{n} x_{1}, 1 \leq i \leq n-1$, with a longest edge removed.

It is easy to see that the conditions are optimal. So if two or more edges are longer than the radius, then the Steiner tree may not run around $C$, as in the theorem.

In this paper we generalize this result to convex configurations. We call a configuration "convex" if the vertices lie on the boundary of a convex polygon. We include the case where an interior angle of the polygon is $180^{\circ}$. We use $X$ 
for the collection $\left\{x_{1}, \ldots, x_{n}\right\}$. Also, indices are taken modulo $n$, so that $x_{n+1}$ denotes $x_{1}$.

Definition. We define the radius of maximum curvature as follows. Any three neighboring vertices $x_{i-1}, x_{i}, x_{i+1}$ describe a unique circle (possibly of infinite radius, i.e., a line) such that they lie on the circle. Now the circle of maximum curvature for a particular configuration $X$ is the smallest such circle for $2 \leq i \leq$ $n+1$. The radius of this circle is called the radius of maximum curvature for $X$. Also, $x_{i}$ is then called a vertex of maximum curvature.

If $X$ satisfies the conditions of Theorem 1, then even without the result of the theorem it can easily be shown that the minimal spanning tree consists of the edges $x_{i} x_{i+1}$ plus $x_{n} x_{1}, 1 \leq i \leq n-1$, with a longest edge removed.

In order to prove a similar theorem for convex configurations we need to ensure the configurations also have the property that the minimal spanning tree consists of edges $x_{i} x_{i+1}$ plus $x_{n} x_{1}, 1 \leq i \leq n-1$, with a longest edge removed. Thus suppose the configuration $X$ is convex with radius of maximum curvature $r$. It is not enough to just assume that at most one of the edges has length strictly greater than $r$. For example we may take the configuration $X$ to be the teardrop (see Fig. 1). Here the diagonal $x_{i-1} x_{i+1}$ may be short and an obvious Steiner vertex inside angle $x_{i-1} x_{i} x_{i+1}$ would be in a Steiner minimal tree.

The "problem" that occurs with the teardrop can best be described by the "compatibility" of the circles through $x_{i-1}, x_{i}, x_{i+1}$ and $x_{i}, x_{i+1}, x_{i+2}$. Suppose an edge $x_{i} x_{i+1}$ has an acute angle $x_{i-1} x_{i} x_{i+1}$ at one end and an obtuse angle $x_{i} x_{i+1} x_{i+2}$ at the other. A condition needed to ensure the spanning tree has no diagonals is that both centers of the circles lie "inside" the edge $x_{i} x_{i+1}$, i.e., lie on the same side of $x_{i} x_{i+1}$ as the rest of the configuration (see Fig. 2).

The case where an edge $x_{i} x_{i+1}$ (necessarily a long edge) has an acute angle at each end also needs consideration-again we want compatibility of the circles. Here the centers of the circles must both be on the same side of $x_{i} x_{i+1}$ (see Fig. 3). If an edge has obtuse angles at each end, then necessarily the circles will be "compatible" (see Fig. 4).

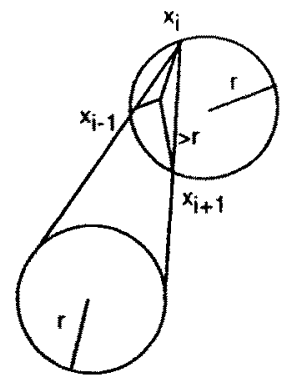

Fig. 1. The teardrop configuration. 


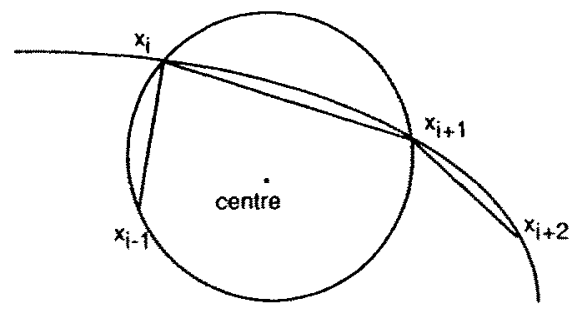

centre

Fig. 2. Compatibility of the circles for an edge with an acute angle on one end and an obtuse angle on the other.
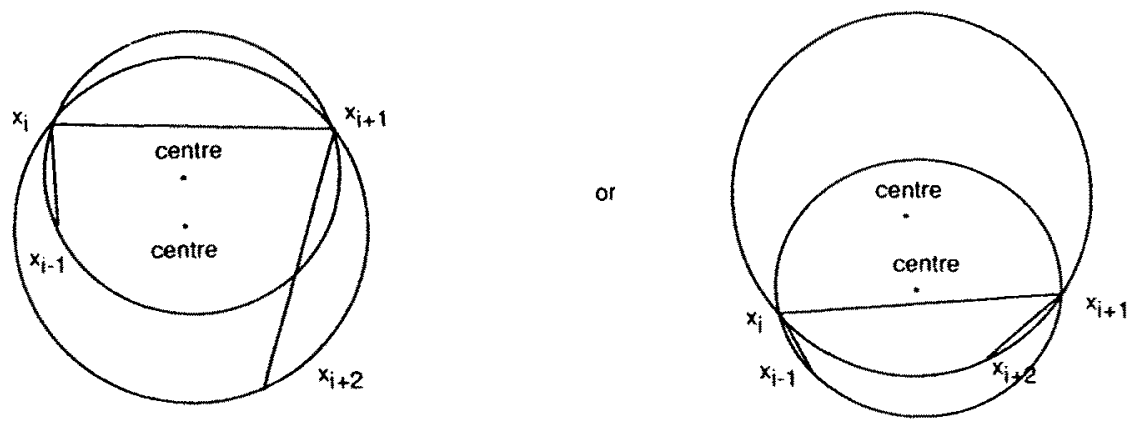

Fig. 3. Compatibility of the circles for an edge with acute angles at each end.

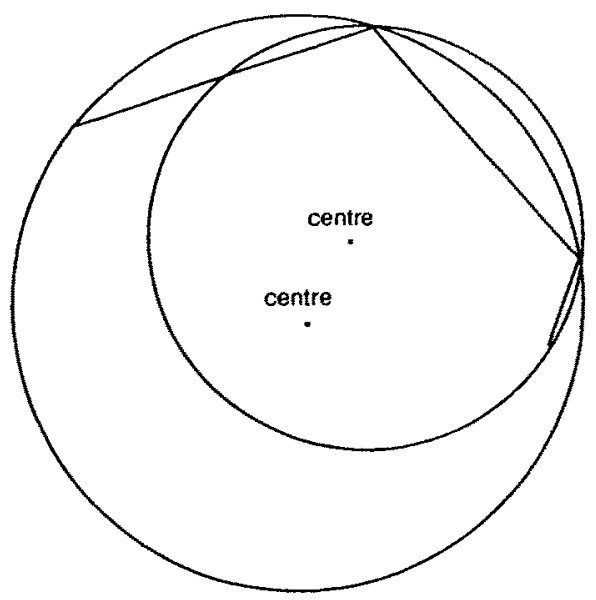

Fig. 4. Compatibility of the circles for an edge with obtuse angles at each end. 
In this paper we prove the following result:

Theorem 2. Suppose that $X=\left\{x_{1}, \ldots, x_{n}\right\}$ is a convex configuration with radius of maximum curvature $r$ with $x_{i-1}$ adjacent to $x_{i}, 2 \leq i \leq n$, and let the circles through $x_{i-1}, x_{i}, x_{i+1}$, and $x_{i} x_{i+1} x_{i+2}$ be compatible. If at most one of the edges $x_{i} x_{i+1}$ and $x_{n} x_{1}$ has length strictly greater than $r$ for $1 \leq i \leq n-1$, then the shortest network $S$ consists of all the edges $x_{i} x_{i+1}$ plus $x_{n} x_{1}, 1 \leq i \leq n-1$, with a longest edge removed. Note that, as a consequence of the proof, any Steiner tree containing vertices other than the $x_{i}$ will be longer.

Let $T$ be the minimal spanning tree for $X . T$ is the smallest tree with endpoints at $X$ and no extra vertices. For $X$ as described in the statement of the theorem it follows from our proof of Theorem 2 that $T$ consists of the edges $x_{i} x_{i+1}$ plus $x_{n} x_{1}$ for $1 \leq i \leq n-1$, with a longest edge deleted. The conditions, assumed in Theorem 2 , on $X$ ensure the angles $x_{i-1} x_{i} x_{i+1} \geq 120^{\circ}$ in the case that neither $x_{i-1}$ nor $x_{i} x_{i+1}$ has length greater than $r$. Of course, if $X$ has several equal longest edges, then there are several choices for $T$. If there is a unique long edge, then it may or may not have length greater than $r$.

Note also that in particular if the vertices lie on a smooth convex curve, curvature $\leq 1 / r$ for some $r$, and if at most one of the edges $x_{i} x_{i+1}$ and $x_{n} x_{1}$ has length strictly greater than $r$ for $1 \leq i \leq n-1$, then we have a configuration satisfying the conditions of the theorem. We give a separate proof in the Appendix that such a configuration has minimal tree $T$ consisting of edges $x_{i} x_{i+1}$ plus $x_{n} x_{1}$ for $1 \leq i \leq n-1$ with a longest edge removed.

\section{The Variational Approach}

We view the problem as a variational one, see [4]-[7]. Let $L_{S}$ (resp. $L_{T}$ ) denote the length of $S$ (resp. $T$ ) and let the Steiner ratio $\rho=L_{S} / L_{T}$. Clearly, if $X$ is any set of points satisfying the conditions of Theorem 2 , it suffices to show that $\rho \geq 1$ and $\rho=1$ only if $S$ and $T$ coincide.

In [4] the differentiability of the Steiner ratio $\rho$ is discussed on the simplex $\Delta$ parametrized by the edges of $S . \rho$ has a differential $D_{\rho}(V)$ in the direction of any vector $V$ at $Y$ in $\Delta$ and is Gateaux or piecewise differentiable. The first variation formula $[4$, Lemma 1$]$ is then

$$
D \rho(V)=\dot{L}_{T} / L_{T}\left(\dot{L}_{S} / \dot{L}_{T}-\rho\right)
$$

where $\dot{L}_{T}$ is the derivative of $L_{T}$ in direction $V$ and similarly for $\dot{L}_{S}$.

Definition. The configuration space $\Delta_{0}$ is the collection of all sets of $n$ points $X=\left\{x_{1}, \ldots, x_{n}\right\}$ forming a convex configuration and satisfying the conditions of Theorem 2 . 
Throughout this paper we work in $\Delta_{0}$ which is the product of a compact set and $(0, \infty)$. By a homothety (rescaling) we can arrange that $X$ has diameter $1 ; \Delta_{0}$ is then homeomorphic to the product of the set of such configurations $X$ and $(0, \infty)$.

A critical point of $\rho$ is a point $Y$ for which $D_{p}(V) \geq 0$ for any vector $V$ at $Y$. If $\dot{L}_{T}<0$ this implies $\dot{L}_{S} / \dot{L}_{T} \leq \rho$.

We argue as follows: Assume $X$ is a minimum configuration for $\rho$, where $S \neq T$ and $L_{S} / L_{T} \leq 1$. Then find some direction $V$ so that $\dot{L}_{T}<0$ and $\dot{L}_{S} / \dot{L}_{T}>1$. From (*) we have $\dot{\rho}=D_{\rho}(V)<0$ contradicting $X$ being a critical point. The case $L_{S} / L_{T}=1$ implies $S=T$ is a consequence of the proof. Note that $\rho$ achieves a minimum since we can rescale so that diameter $X=1$ without altering $\rho$.

\section{Proof of Theorem 2}

Let $X$ be a convex configuration satisfying the conditions of Theorem 2 . Let $T$ be the spanning tree for $X$ consisting of all edges $x_{i-1} x_{i}$ and $x_{n} x_{1}$ with a longest edge removed. Note that, by assumption, all edges of $T$ have length at most $r$. We consider the following cases.

Case I. Assume there is some vertex $x_{k}$ with neighbors $x_{k-1}$ and $x_{k+1}$, neither of which are vertices of maximum curvature. This assumption is needed as the variation at $x_{k}$ increases the curvature at $x_{k-1}$ and $x_{k+1}$. The other situation is dealt with later. Now suppose neither $x_{k-1} x_{k}$ nor $x_{k} x_{k+1}$ has length greater than $r$. Then angle $x_{k-1} x_{k} x_{k+1}$ is obtuse and in fact at least $120^{\circ}$. Suppose it is less than $180^{\circ}$. There are two possibilities for the Steiner tree at $x_{k}$.

Variation 1 , Variation 1 covers the situation where there is one Steiner edge at $x_{k}$. If $\alpha($ resp. $\beta)$ is the angle between $x_{k-1} x_{k}$ (resp. $\left.x_{k} x_{k+1}\right)$ and $S$ at $x_{k}$ do a variation along $S$ (called a "shrink") at $x_{k}$, see Fig. 5 . $\left|\dot{L}_{S}\right|=1,\left|\dot{L}_{T}\right|=\mid \cos \alpha+$ $\cos \beta \mid$ if $x_{k-1} x_{k}$ and $x_{k} x_{k+1}$ are in $T$, hence $\dot{L}_{S} / \dot{L}_{T} \geq 1$. Note $\alpha+\beta \geq 120^{\circ}$ implies $|\cos \alpha+\cos \beta| \leq 1$. If, say, $x_{k-1} x_{k}$ is not in $T$ we have $\left|\dot{L}_{T}\right|=|\cos \beta|$, hence $\mid \dot{L}_{s} / \dot{L}_{T}>1$, if $\beta<90^{\circ}$. However, if $\beta \geq 90^{\circ}$ do a variation at $(90-\varepsilon)^{\circ}$ to $x_{k} x_{k+1}$. Since angle $x_{k-1} x_{k} x_{k+1}<180^{\circ}$ we can always choose $\varepsilon$ so that $\left|\dot{L}_{S}\right|>\left|\dot{L}_{T}\right|$ and $\dot{L}_{S} / \dot{L}_{T}>1$. Choosing the variation at an angle just less than $90^{\circ}$ ensures $x_{k} x_{k+1}$ does not increase in length (allowing $x_{k} x_{k+1}$ to have length $r$ ). If $\alpha=0$, say, then $\beta \geq 120^{\circ}$, so we can do a $(90-\varepsilon)^{\circ}$ variation. In fact if $S$ and $T$ coincide $\dot{L}_{S} / \dot{L}_{T}=1$. If $L_{S} / L_{T}<1$, then this variation decreases the ratio. If $L_{S} / L_{T}=1$, this variation means the ratio remains constant up to first derivatives.

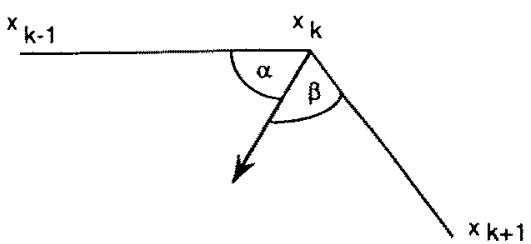

Fig. 5. The shrink variation. 


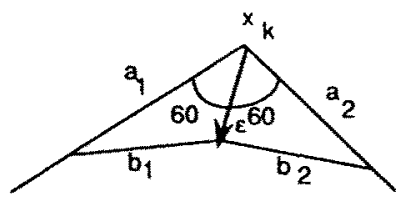

Fig. 6. The variation for an angle of $120^{\circ}$.

If $\alpha, \beta$ are not both equal to $60^{\circ}$, then $|\cos \alpha+\cos \beta|<1$ so $\dot{L}_{S} / \dot{L}_{T}>1$. Now suppose the angle $x_{k-1} x_{k} x_{k+1}$ is $120^{\circ}$ and $\alpha=\beta=60^{\circ}$. Do a shrink at $x_{k}$ by moving $x_{k}$ along $S$ a distance $\varepsilon$. See Fig. 6 .

The Steiner ratio for the new configuration will be

$$
\left(L_{S}-\varepsilon\right) /\left(L_{T}-\left(a_{1}+a_{2}\right)+b_{1}+b_{2}\right) .
$$

Here $b_{i}=\sqrt{a_{i}^{2}+\varepsilon^{2}-a_{i} \varepsilon}$ for $i=1,2$, by the cosine rule in a $60^{\circ}$ triangle. This is less than $L_{S} / L_{T}$, the ratio before the variation is made and which was chosen to be a minimum, if $a_{1}+a_{2}-b_{1}-b_{2}<\varepsilon$.

We show

$$
a_{1}+a_{2}-\sqrt{a_{1}^{2}+\varepsilon^{2}-a_{1} \varepsilon}-\sqrt{a_{2}^{2}+\varepsilon^{2}-a_{2} \varepsilon}<\varepsilon .
$$

The terms involving $a_{1}, a_{2}$ are independent.

$$
\begin{aligned}
a_{1}-a_{1} \sqrt{1+\left(\frac{\varepsilon}{a_{1}}\right)^{2}-\left(\frac{\varepsilon}{a_{1}}\right)} & =a_{1}-a_{1}\left(1+\left(\frac{\varepsilon^{2}}{a_{1}^{2}}-\frac{\varepsilon}{a_{1}}\right)\right)^{1 / 2} \\
& =a_{1}-a_{1}\left(1+\frac{1}{2}\left(\frac{\varepsilon^{2}}{a_{1}^{2}}-\frac{\varepsilon}{a_{1}}\right)-\frac{1}{8}\left(\frac{\varepsilon^{2}}{a_{1}^{2}}-\frac{\varepsilon}{a_{1}}\right)^{2} \cdots\right) \\
& =-\frac{3 \varepsilon^{2}}{8 a_{1}}+\frac{\varepsilon}{2} \quad \text { up to second order. }
\end{aligned}
$$

Similarly for $a_{2}$. We have $\varepsilon-3 \varepsilon^{2} / 8 a_{1}-3 \varepsilon^{2} / 8 a_{2}+$ higher-order terms $<\varepsilon$ for $\varepsilon$ small. So $L_{S} / L_{T}$ does actually decrease if we do the shrink at $x_{k}$ giving a contradiction.

Variation 2. Variation 2 covers the case where two edges of the Steiner tree meet at $x_{k}$. Here we do a variation along the bisector of the angle $2 \delta$ of $S$ which is at least $120^{\circ}$, see Fig. 7. If $\beta$ (resp. $\gamma$ ) is the angle between $x_{k-1} x_{k}$ (resp. $x_{k} x_{k+1}$ ) and the nearest edge of $S$,

$$
\begin{aligned}
& \dot{L}_{S}=-2 \cos \delta, \\
& \dot{L}_{T}=-\cos (\delta+\beta)-\cos (\delta+\gamma) \text { or } \quad \dot{L}_{T}=-\cos (\delta+\beta)
\end{aligned}
$$

if $x_{k} x_{k+1}$ is not in $T$. 


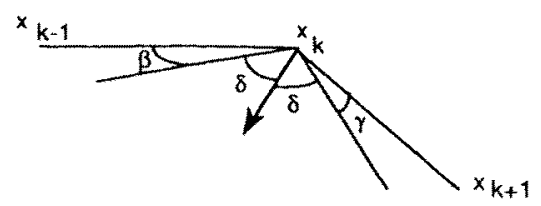

Fig. 7. This variation bisects the angle.

So $\dot{L}_{S} / \dot{L}_{T}>1$. This also covers the case where $\gamma($ and/or $\beta$ ) may be zero. In fact if $S$ and $T$ coincide $\dot{L}_{S} / \dot{L}_{T}=1$ and the same remark holds as in Variation 1 . Note that Variations 1 and 2 never increase the ratio nor do they increase the length of any $x_{i} x_{i+1}$.

Suppose angle $x_{k-1} x_{k} x_{k+1}$ is less than $120^{\circ}$. Then either $x_{k-1} x_{k}$ or $x_{k} x_{k+1}$ has length greater than $r$. We cannot have two edges of the Steiner tree at $x_{k}$, as the angle is less than $120^{\circ}$. If the Steiner tree is as in Fig. 5 we can do the variation described in Variation 1 when $x_{k-1} x_{k}$, say, is not in $T$. Again, if $S$ and $T$ coincide a similar remark holds as discussed in Variation 1.

Suppose we do not have a vertex $x_{i}$ where either Variation 1 or 2 decreases the ratio straight away. Our argument continues by induction on the number of vertices of maximum curvature.

Case II. If there is only one vertex $x_{\mathrm{i}}$ of maximum curvature, then we can use Variation 1 or 2 easily. Since $S \neq T$ there will be at least three edges of $S$ not in $T$ which end at three different $x_{j}$ and at least one of the $x_{j}$ 's will have neighbors of nonmaximum curvature.

The only case not covered is the situation where the three Steiner edges end at vertices of $180^{\circ}$ angles. Variation 1 or 2 would result in a nonconvex configuration. In this event we can do the following at one of these vertices $x_{j}$. If a vertex, say $x_{j-1}$, neighboring $x_{j}$ is less than $180^{\circ}$ do Variation 1 or 2 at $x_{j-1}$ which decreases the angle at $x_{j}$ and then we again use Variation 1 or 2 at $x_{j}$ decreasing the ratio. However, in case both neighbors of $x_{j}$ are $180^{\circ}$, choose the closest vertex, $x_{j-k}$ say, to $x_{j}$ which is not $180^{\circ}$. Pivot the arc $x_{j} x_{j-1} \cdots x_{j-k}$ around $x_{j}$ which does not increase $S$ or $T$ along the arc but decreases the angle at $x_{j}$. Now do Variation 1 or 2 at $x_{j}$ and decrease the ratio.

Lemma. Suppose $x_{i}, \ldots, x_{j}$ are neighboring vertices all with maximum curvature, then they are all cocircular.

Proof. Note that the compatibility of the circles through three neighboring vertices implies the centers lie on the same side of $x_{i}, \ldots, x_{j}$. The proof uses elementary geometry.

Case III. Suppose there are neighboring vertices $x_{i}, \ldots, x_{i+m}$ all with maximum curvature. By the lemma they are cocircular and suppose the length of the circular arc joining them is greater than $\pi r$. Then all the vertices $x_{1}, \ldots, x_{n}$ lie on the same circle. This follows since if $x_{i-1}$, say, lies inside the circle the curvature at $x_{i}$ will be greater than $r$. If $x_{i-1}$ lies outside the circle, then by convexity it must lie 


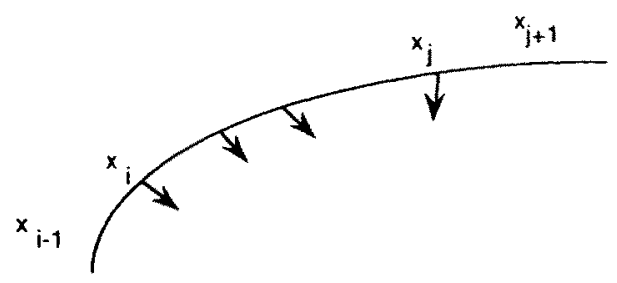

Fig. 8. Decreasing the curvature on the arc.

between the circle and the tangents to the circle at $x_{i}$ and $x_{i+m}$ or possibly on the tangents. In either case there will be a vertex, see Fig. 10(b), with curvature greater than $1 / r$, a contradiction.

Case IV. Choose an arc of the configuration where the vertices are of maximum curvature and lie within a semicircle. The idea is to move these vertices so that:

(i) The ratio is nonincreasing.

(ii) The distances between vertices do not increase, so as to preserve the conditions of the theorem.

In Fig. 8 the vertices $x_{i}, \ldots, x_{j}$ have maximum curvature. The vertices $x_{i-1}, x_{j+1}$ are not of maximum curvature. Thus we can decrease angles in the spanning tree $T$ at $x_{i-1}, x_{j+1}$. Do Variations 1 and 2 at $x_{i}, \ldots, x_{j}$ depending on the Steiner tree $S$ at each vertex. If we keep $x_{i-1}, x_{j+1}$ fixed we can just flatten the circle between these vertices by adjusting the speed of the variation at each of $x_{i}, \ldots, x_{j}$. One convenient way of achieving this is to choose circular arcs of increasing radius through $x_{i-1}$ and $x_{j+1}$ and to move $x_{i}, \ldots, x_{j}$ so they lie on such ares. Note we must have the length of the circle between $x_{i-1}$ and $x_{j+1}$ to be at most $\pi r$ to flatten the circular arc. This is the same as decreasing the curvature at $x_{i}, \ldots, x_{j}$. If $S \neq T$ somewhere between $x_{i}$ and $x_{j}$, say at $x_{k}$, the ratio will actually decrease. If $S=T$ here but $L_{S} / L_{T}<1$, again the ratio decreases. This contradicts the minimality of the ratio. In any event we have decreased the number of vertices with maximum curvature and the proof can continue by induction. In effect we are making the configuration more circular by decreasing the curvature along these arcs of maximum curvature and length less than $\pi r$.

\section{Acknowledgment}

We would like to thank the referees for a number of helpful comments.

\section{Appendix}

The proposition below is independent of the results above. 
Proposition. Suppose $X=\left\{x_{1}, \ldots, x_{n}\right\}$ is a convex configuration lying on a smooth curve with curvature $\leq 1 / r$. If at most one of the edges $x_{i} x_{i+1}$ and $x_{n} x_{1}$ has length strictly greater than $2 r$, for $1 \leq i \leq n-1$, then the minimal tree $T$ consists of the edges $x_{i} x_{i+1}$ plus $x_{n} x_{1}$ for $1 \leq i \leq n-1$ with a longest edge deleted.

Proof. Suppose $x_{i} x_{j} \in T$, where $j \neq i+1, i-1$. Consider the angles $\alpha, \beta$ between $x_{i} x_{j}$ and the tangents to the convex curve $\Gamma$ described by $X$. See Fig. 9(a). Assume first that $\alpha$, the angle at $x_{i}$, say, is obtuse and $\beta$ is acute. See Fig. $9($ b). Let $n(x)$ be the normal from $x$ orthogonal to some tangent to $\Gamma$. Consider $n\left(x_{j}\right)$. We can shorten $x_{i} x_{j}$ by moving $x_{i}$ in the direction $\pi-\alpha$ toward $n\left(x_{j}\right)$. If we reach a point $x$, before we reach $n\left(x_{j}\right)$ such that $n(x)$ meets $\Gamma$ at $x_{j}$, then we stop there since we have found a normal which is shorter than $x_{i} x_{j}$. See Fig. $9\left(\right.$ b). Otherwise $n\left(x_{j}\right)$ is shorter than $x_{i} x_{j}$.

Suppose next that both angles $\alpha, \beta$ are acute. We argue that either $x_{i} x_{j}$ is longer than some normal or $x_{i} x_{j}$ is longer than all the edges $x_{i} x_{i+1}$, $x_{i+1} x_{i+2}, \ldots, x_{j-1} x_{j}$. The latter gives a contradiction, since then $x_{i} x_{j}$ is not in $T$.

The argument is by induction on $|i-j| \geq 2$. Start to shrink $x_{i} x_{j}$ by moving $x_{i}$ on the $\alpha$ side. There are two cases. Firstly, if $x_{i}$ reaches $x_{i+1}$, then $\left|x_{i+1} x_{j}\right|<\left|x_{i} x_{j}\right|$. By induction, $\left|x_{i+1} x_{j}\right| \leq\left|x_{i+1} x_{i+2}\right|,\left|x_{i+2} x_{i+3}\right|, \ldots$. Similarly, by deforming $x_{j}$ in direction $\beta$, we get $\left|x_{i} x_{j-1}\right|<\left|x_{i} x_{j}\right| \leq\left|x_{i} x_{i+1}\right|,\left|x_{i+1}, x_{i+2}\right|, \ldots$. So the result

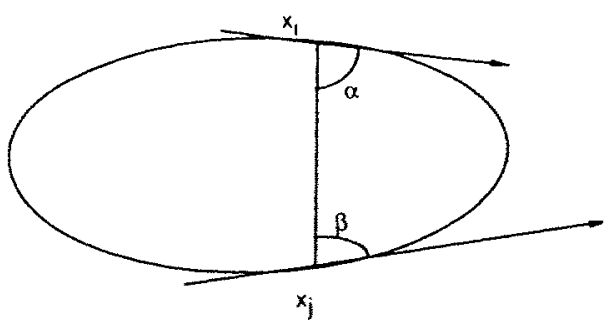

(a)

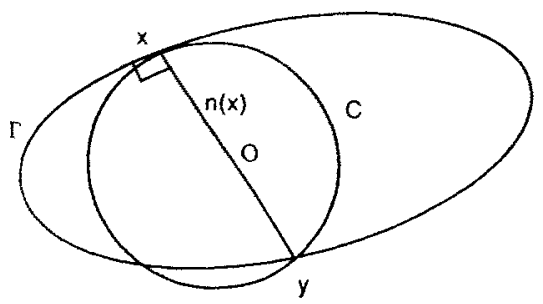

(c)

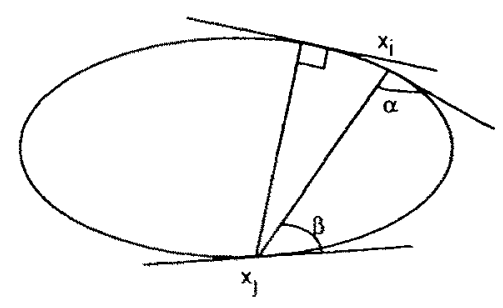

(b)

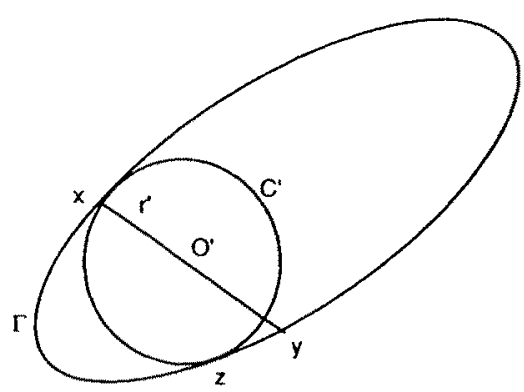

(d)

Fig. 9. Comparing a diagonal edge with a shortest normal. 


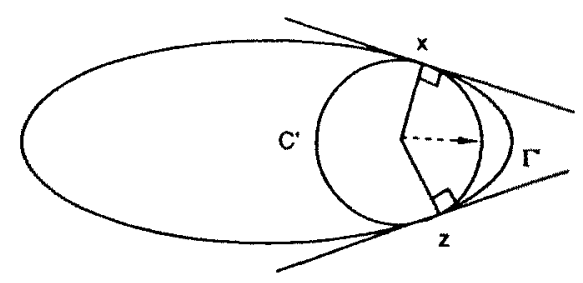

(a)

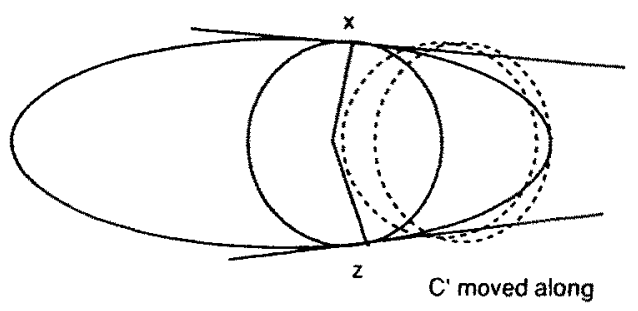

(b)

Fig. 10. Shifting the circle.

follows. In the second case, there is a point $x$ between $x_{i}$ and $x_{i+1}$ with $n(x)$ meeting $\Gamma$ at $x_{j}$. This gives the claim.

Let $|n(x)|$ be the length of the normal, let $d$ be the shortest normal, and let $r$ be the smallest radius of curvature. We show $|n(x)| \geq d \geq 2 r$. Draw a circle $C$ centred at the midpoint of $n(x)$ through $x, y$ the ends of $n(x)$. See Fig. 9(c). Shrink $C$ to $C^{\prime}$ until it touches the curve at some point $z$ but still passes through $x$ and has centre on $n(x)$. See Fig. $9(d)$. Let us assume $|n(x)|<2 r$ and get a contradiction. Then radius $C^{\prime}=r^{\prime} \leq \frac{1}{2}|n(x)|<r$. So $C^{\prime}$ lies inside the curve at $x$ as the curvature is greater than that of $\Gamma$. The same picture is true at $z$. Without loss of generality $C^{\prime}$ is so small that it does not cross the curve anywhere (otherwise shrink $C^{\prime}$ and get a new $z$ ).

Assume we are in the situation in Fig. 10(a). By convexity, the part of the curve $\Gamma^{\prime}$ between $x$ and $z$ lies between the tangents at $x$ and $z$. Now shift $C^{\prime}$ along the line bisecting the radii to $x$ and $z$. Then the shifted circle cuts $\Gamma^{\prime}$ at two more points as shown. Eventually these two points will coalesce so the shifted $C^{\prime}$ is tangent to $\Gamma^{\prime}$ here. See Fig. 10(b). However then $\Gamma^{\prime}$ has a point with curvature greater than $r^{\prime}$, a contradiction. Note that we choose to move $C$ toward the arc $\Gamma^{\prime}$ of greatest curvature.

\section{References}

1. M. R. Garey, R. L. Graham, and D. S. Johnson, The Complexity of Computing Steiner Minimal Trees, SIAM J. Appl. Math. 32 (1977), 835-859.

2. R. L. Graham, Some Results on Steiner Minimal Trees, Unpublished manuscript, 11 May 1967.

3. Z. A. Melzak, On the Problem of Steiner, Canad. Math. Bull. 4 (1961), 143-148. 
4. J. H. Rubinstein and D. A. Thomas, A Variational Approach to the Steiner Network Problem, Ann. Oper. Res. 33 (1991), 481-499.

5. J. H. Rubinstein and D. A. Thomas, The Steiner Ratio Conjecture for Six Points, J. Combin. Theory Ser. A 58 (1991), 54-77.

6. J. H. Rubinstein and D. A. Thomas, The Steiner Ratio Conjecture for Cocircular Points, Discrete Comput. Geam. 7 (1992), $77-86$.

7. J. H. Rubinstein and D. A. Thomas, Graham's Problem on Shortest Networks for Points on a Circle, Algorithmica 7 (1992), 193-218.

Received July 9, 1991, and in revised form March 19, 1992. 